\title{
Pneumococcal 13-valent Conjugate Vaccine
}

National Cancer Institute

\section{Source}

National Cancer Institute. Pneumococcal 13-valent Conjugate Vaccine. NCI Thesaurus.

Code $C 97121$.

A pneumococcal conjug ate vaccine containing 13 different strains of the bacterium Streptococcus pneumoniae, used in children and studied in immunocompromised patients for the prevention of pneumococcal disease. The pneumococcal 13-valent conjug ate vaccine contains capsular antigen polysaccharides derived from the S. pneumoniae serotypes 1, 3, 4, 5, 6A, 6B, 7F, 9V, 14, 18C, 19A, 19F and 23F that are individually conjugated to a nontoxic diphtheria cross-reactive material (CRM) carrier protein (CRM197). Upon vaccination, pneumococcal 13-valent conjugate vaccine induces active immunization against 13 different serotypes of S. pneumoniae and protects against pneumococcal disease. 